\title{
IgG subclass staining in renal biopsies with membranous glomerulonephritis indicates subclass switch during disease progression
}

\author{
Cheng Cheng Huang ${ }^{1}$, Amy Lehman ${ }^{2}$, Alia Albawardi ${ }^{1}$, Anjali Satoskar ${ }^{1}$, Sergey Brodsky ${ }^{1}$, \\ Gyongyi Nadasdy ${ }^{1}$, Lee Hebert ${ }^{3}$, Brad Rovin ${ }^{3}$ and Tibor Nadasdy ${ }^{1}$ \\ ${ }^{1}$ Department of Pathology and Laboratory Medicine, The Ohio State University, Columbus, OH, USA; ${ }^{2}$ Center \\ for Biostatistics, The Ohio State University, Columbus, OH, USA and ${ }^{3}$ Department of Medicine, The Ohio \\ State University, Columbus, $\mathrm{OH}$, USA
}

\begin{abstract}
Recent breakthrough findings revealed that most patients with idiopathic (primary) membranous glomerulonephritis have IgG4 antibodies to the phospholipase A2 receptor (PLA2R). These IgG4 antibodies can be detected in the glomerular immune complexes and they colocalize with PLA2R. In secondary forms of membranous glomerulonephritis, such IgG4 antibodies are absent or less prevalent. There are no studies addressing the IgG subclass distribution across different stages of membranous glomerulonephritis. During a 25-month period, we identified 157 consecutive biopsies with membranous glomerulonephritis with adequate tissue for light, immunofluorescence and electron microscopy. Of the 157 membranous glomerulonephritis cases, 114 were primary membranous glomerulonephritis and 43 were secondary membranous glomerulonephritis. We compared the intensity of IgG subclass staining (on a semiquantitative scale of 0 to $3+$ ) and the IgG subclass dominance between primary and secondary membranous glomerulonephritis and between the different stages of membranous glomerulonephritis. In primary membranous glomerulonephritis most ( $76 \%$ of cases) were IgG4 dominant. In contrast, in secondary membranous glomerulonephritis IgG1 was dominant in $60 \%$ of biopsies $(P=0.0018)$. Interestingly, in early stage (stage 1$)$ primary membranous glomerulonephritis, IgG1 was the dominant IgG subclass (64\% of cases); in all later stages IgG4 dominated $(P=0.0493)$. It appears that there is an inverse relationship between the intensity of glomerular capillary IgG4 and C1q staining. In secondary forms of membranous glomerulonephritis (heterogeneous group with low case numbers), we did not find such associations. Our data indicate that in early stage membranous glomerulonephritis, antibody response is different from later stages, with IgG1 dominant deposits. It is possible that early on, antigens other than PLA2R have an important role, Alternately, there may be an IgG subclass switch in the antibody response with IgG4 taking over later as the dominant immunoglobulin.
\end{abstract}

Modern Pathology (2013) 26, 799-805; doi:10.1038/modpathol.2012.237; published online 18 January 2013

Keywords: IgG subclass; membranous glomerulonephritis

Membranous glomerulonephritis is a unique form of immune complex glomerulonephritis manifested by glomerular subepithelial IgG-containing immune complex deposits and usually heavy proteinuria. In most patients, the etiology is unknown; therefore, this form of membranous glomerulonephritis used to be designated as idiopathic membranous

Correspondence: Dr T Nadasdy, MD, Department of Pathology and Laboratory Medicine, The Ohio State University, 320 West 10th Avenue, M018 Starling-Loving Hall, Columbus, OH 43210, USA.

E-mail: tibor.nadasdy@osumc.edu

Received 4 September 2012; revised 14 December 2012; accepted 14 December 2012; published online 18 January 2013 glomerulonephritis. Occasionally, an underlying disease process or condition, that can be associated with membranous glomerulonephritis, is present, such as systemic lupus erythematosus and other autoimmune diseases, malignancy, certain chronic infections (eg, hepatitis B hepatitis C, syphilis), heavy metal exposure (such as gold or mercury), drugs (eg, penicillamine, non-steroidal anti-inflammatory drugs) or sarcoidosis. ${ }^{1}$ Such cases of membranous glomerulonephritis are referred to as secondary membranous glomerulonephritis, and if the underlying cause (eg, chronic infection, toxin, drug, malignancy) can be eliminated, the membranous glomerulonephritis can be cured. In contrast, in idiopathic membranous glomerulonephritis, the 
underlying cause could not be treated and the patients either had persistent disease with proteinuria and slow progression or recurrences after remissions.

Recently, we have witnessed an important new development in the research on the pathogenesis of membranous glomerulonephritis. Beck and coworkers $^{2}$ identified phospholipase A2 receptor (PLA2R) as a potential target antigen in idiopathic (now more appropriately called primary) membranous glomerulonephritis. PLA2R is a membrane glycoprotein localized to the podocytes. In their original report, Beck et $a l^{2}$ found that $70 \%$ of patients with idiopathic/primary membranous glomerulonephritis had circulating IgG4 antibodies to PLA2R. No anti-PLA2R was detected in control patients including patients with secondary forms of membranous glomerulonephritis. In biopsies with idiopathic/primary membranous glomerulonephritis, the staining for PLA2R colocalized with IgG (specifically IgG4) in the glomerular subepithelial deposits. ${ }^{2}$ These data were later confirmed by other studies. ${ }^{3,4}$ However, PLA2R may not be the only target antigen in idiopathic membranous glomerulonephritis, because other recent studies described potential target antigens such as aldose reductase, superoxide dismutase, ${ }^{5} \alpha$ enolase ${ }^{6}$ and bovine serum albumin, ${ }^{7}$ however, with less prevalence. ${ }^{8}$ Many patients with primary membranous glomerulonephritis have several types of circulating anti-podocyte antibodies at the same time but, among these antibodies, anti-PLA2R antibodies are the most prevalent. $^{8}$ These antibodies are primarily of the IgG4 subclass, which is consistent with the well-known finding that, in idiopathic membranous glomerulonephritis, the subepithelial immune complexes contain mostly IgG4. In contrast, several papers indicate that in secondary forms of membranous glomerulonephritis, the IgG subclass distribution is different from the primary form, and the deposits are frequently not IgG4 dominant. ${ }^{9-16}$ However, even idiopathic membranous glomerulonephritis is a heterogeneous disease if we consider the morphological appearance of the glomeruli during disease progression. Membranous glomerulonephritis, by pathological criteria, is divided into four stages according to Ehrenreich and Churg. ${ }^{17}$ In stage I membranous glomerulonephritis, the subepithelial immune complex deposits are not separated by glomerular basement membrane (GBM) spikes, indicating recent formation of immune complex deposits (early stage of the disease). In later stages of membranous glomerulonephritis, spike formation becomes increasingly prominent, and eventually the deposits are completely incorporated into the GBM, and many of them may become partially or completely reabsorbed (electron lucent deposits). It is plausible that the morphological evolution of the glomerular capillary deposits in membranous glomerulonephritis reflects changes in the composition of the deposits. Our work tests this hypothesis by analyzing IgG subclass distribution within the deposits in different stages of membranous glomerulonephritis.

\section{Materials and methods}

From January 2007, we began routinely staining every membranous glomerulonephritis biopsy with antibodies to the IgG subclasses. The work encompasses adult renal biopsies from January 2007 to February 2010 with the diagnosis of membranous glomerulonephritis. Cases of membranous glomerulonephritis with superimposed proliferative lesions (such as proliferative lupus nephritis combined with membranous glomerulonephritis or rare cases of crescentic membranous glomerulonephritis) were not included. Only biopsies with glomeruli available for light microscopy, immunofluorescence and electron microscopy were included. During the study period, we identified 157 such biopsies from 157 patients with membranous glomerulonephritis. In 114 patients, the membranous glomerulonephritis was classified as idiopathic. In 43 patients, the membranous glomerulonephritis was classified as secondary because of the following underlying conditions: 15 patients had systemic lupus erythematosus, six patients had an autoimmune disease other than systemic lupus erythematosus (two patients had antiphospholipid antibodies with lupus-like features, two had Sjögren syndrome, one had IgG4 disease, and one had mixed connective tissue disease), ten patients had malignant neoplasm, five patients had hepatitis B infection, five patients had hepatitis $\mathrm{C}$ infection, one patient had sarcoidosis, and one patient had syphilis.

Tissue for light microscopy was routinely fixed in formalin and embedded in paraffin. The paraffin sections were cut at $3 \mu \mathrm{m}$ and stained routinely with hematoxylin and eosin, PAS, trichrome, and Jones methenamine silver stains. Direct immunofluorescence was performed on frozen sections with antibodies to IgG, IgA, IgM, $\kappa$ - and $\lambda$-light chains, C1q, C3, fibrinogen and albumin (Kent Laboratories, kentlabs.com). Direct immunofluorescence with antibodies to IgG1, IgG2, IgG3 and IgG4 (The Binding Site, bindingsite.com) was performed as described previously. ${ }^{18}$ Tissue was processed for electron microscopy according to routine methodology using Spurr's resin for embedding, osmium tetroxide postfixation and uranyl acetate contrasting.

Immunofluorescence staining intensity was semiquantitatively scored during the routine biopsy evaluation from 0 to $3+$ ( 0 negative, $1+$ weak staining, $2+$ moderate staining, $3+$ strong staining). Staining for the IgG subclasses was categorized as predominant if immunofluorescence was clearly 
stronger for one IgG subclass relative to the other three. Immunofluorescence was codominant if one or two (rarely three) antibodies to the various IgG subclasses showed similar staining intensity. For statistical purposes, predominant and codominant stains were lumped together; therefore, in individual groups, the IgG predominance/codominance for the different IgG subclasses adds up to over $100 \%$.

For the ultrastructural staging of membranous glomerulonephritis, we used the Ehrenreich and Churg classification ${ }^{17}$ but our study groups were modified slightly. To study a true, early stage membranous glomerulonephritis group, we considered stage I disease-only biopsies with no spike formation between the subepithelial deposits. If segmental spike formation was evident, we classified membranous glomerulonephritis as stage I-II. Stage II was diagnosed if spike formation was diffuse with only a few deposits incorporated into the GBM. There were relatively few pure stage II diseases; therefore, we introduced a stage II-III group. We diagnosed membranous glomerulonephritis stage II-III if many deposits were already incorporated into the thickened GBM, but there were still numerous subepithelial deposits with spike formation. True stage III membranous glomerulonephritis cases with mostly intramembranous immune complex deposits with only partial resorption and true stage IV cases with mostly reabsorbed immune complex deposits with widespread electron lucencies within the thickened GBM were rare; therefore, we lumped these together into one group (stages III-IV (advanced-stage membranous glomerulonephritis)). This ultrastructural staging was performed retrospectively on every biopsy by two renal pathologists (TN and AA) blinded to the immunofluorescence results.

For each IgG subclass, we first compared the proportions of predominant/codominant samples between primary and secondary membranous glomerulonephritis using $\chi^{2}$ tests. Next, the odds of IgG predominance or codominance between primary and secondary membranous glomerulonephritis, along with 95\% confidence intervals were calculated using univariable logistic regression models. Similarly, the proportions of predominant/codominant samples among different electron microscopy stages or C3, C1q-staining intensity levels were first compared using $\chi^{2}$ tests or Fisher's exact test, where appropriate. Trends in predominance/codominance then explored using Cochran-Armitage tests for trend. All analyses were performed using SAS/ STAT software, v9.2 (SAS Institute Inc., Cary, NC, USA).

\section{Results}

Consistent with previous reports, we found that glomerular capillary deposits were significantly more commonly IgG4 predominant or codominant in primary/idiopathic membranous glomerulonephritis (Figure 1) than in secondary membranous glomerulonephritis (Table 1). From a logistic regression model, the odds of IgG4 predominance or codominance are 3.22 times higher for patients with primary membranous glomerulonephritis compared with secondary membranous glomerulonephritis (95\% confidence intervals:1.54, 6.70; $P=0.0018$ ).

It was unexpected that, in early stage (stage I) of primary membranous glomerulonephritis, the glomerular capillary deposits were IgG1 predominant or codominant in most cases (Figure 2): 14 patients (64\%) of stage I membranous glomerulonephritis had IgG1 predominance or codominance (Table 2, Figure 3). In all later stages of primary membranous glomerulonephritis, IgG4 was the dominant IgG in the glomerular deposits (Figure 1). There appears to be a borderline significant difference in the proportions of patients with IgG1 predominance/codominance among the different glomerular stages
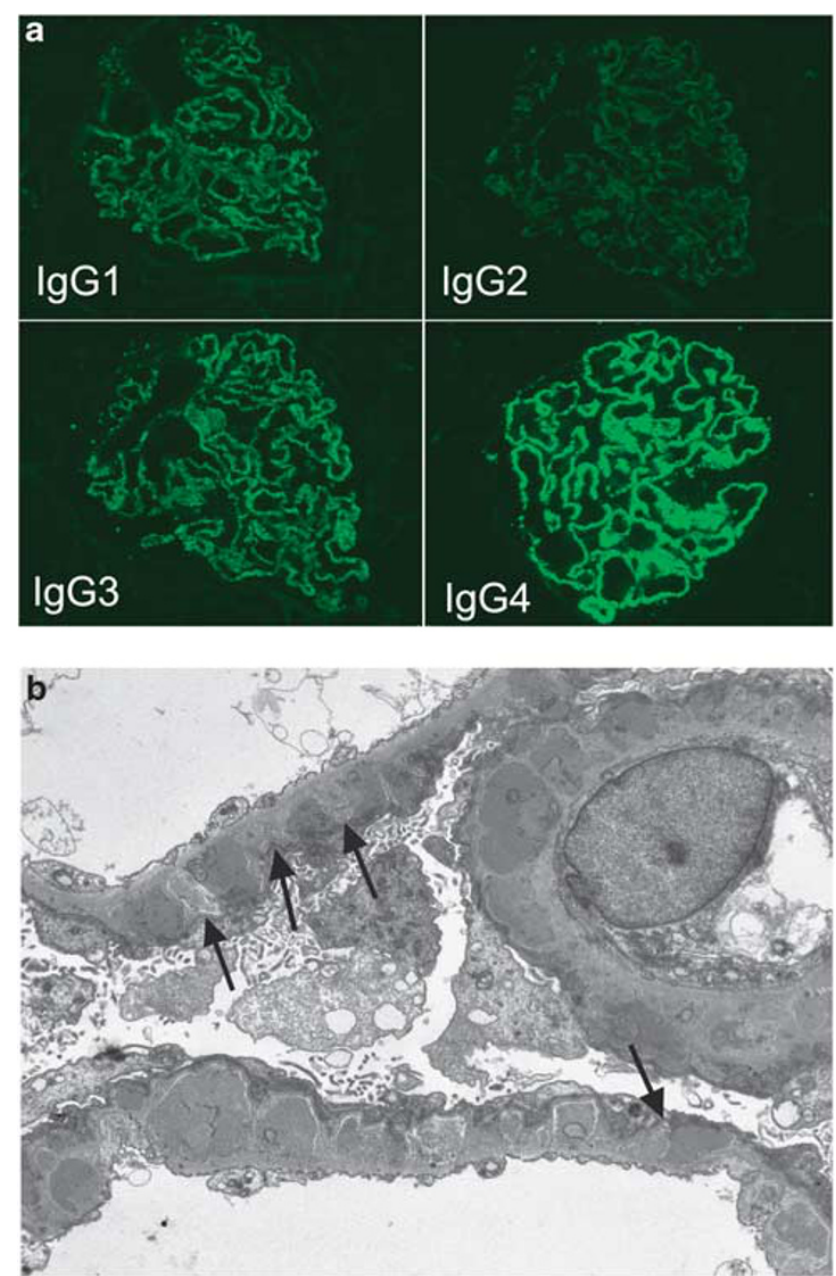

Figure 1 Primary membranous glomerulonephritis, glomerular stage 2-3. (a) The glomerular capillary deposits are IgG4 dominant. Direct immunofluorescence, $\times 200$. (b) Electron microscopy demonstrates subepithelial and intramembranous electron dense deposits with spike formation (arrows) around the subepithelial deposits. Uranyl acetate-lead citrate, $\times 8000$. 
Table 1 IgG predominance/codominance in primary and secondary membranous glomerulonephritis

\begin{tabular}{lccc}
\hline \multirow{2}{*}{ IgG subclass } & $\mathrm{N}(\%)$ predominance/codominance & \multirow{2}{*}{ P-value } \\
\cline { 2 - 3 } & Primary (n=114) & Secondary (n=43) & \\
\hline IgG1 & $53(46 \%)$ & $26(60 \%)$ & 0.1184 \\
IgG2 & $3(3 \%)$ & $2(5 \%)$ & 0.6151 \\
IgG3 & $20(18 \%)$ & $10(23 \%)$ & 0.4169 \\
IgG4 & $86(75 \%)$ & $21(49 \%)$ & 0.0018 \\
\hline
\end{tabular}
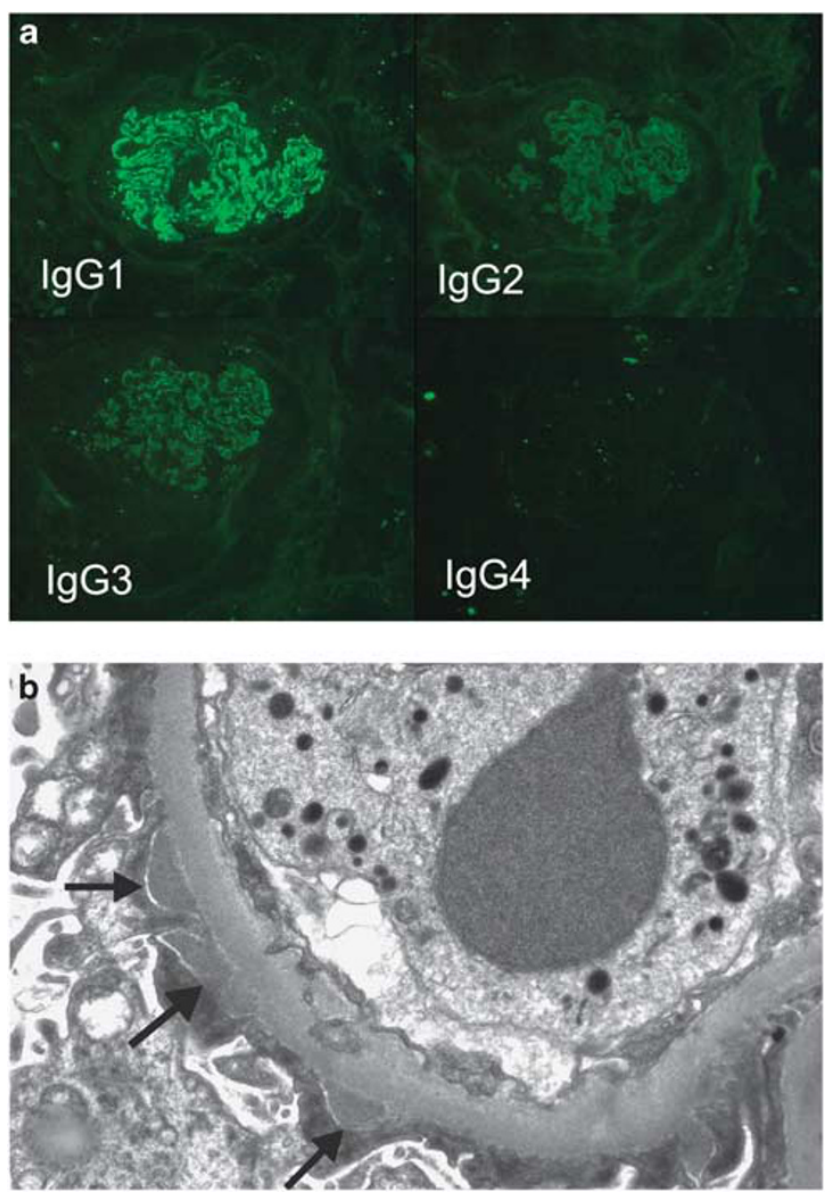

Figure 2 Glomerular stage 1 primary membranous glomerulonephritis. (a) IgG1-dominant glomerular capillary deposits. Direct immunofluorescence, $\times 200$. (b) Electron microscopy shows subepithelial electron dense deposits (arrows) along the glomerular capillary with no spike formation around them. Uranyl acetate-lead citrate $\times 10000$

$(P=0.0493)$ (Table 2). In secondary forms of membranous glomerulonephritis, IgG1 predominance/ codominance was more common than IgG4 codominance/predominance; however, these differences did not reach statistical significance, possibly because of the low case numbers (data not shown).

We also compared the intensity of staining between the different stages of primary membranous glomerulonephritis. In stage I membranous glomer- ulonephritis, the staining intensity, in general, was strongest for IgG1 followed by IgG4 (Table 3). In all other stages, IgG4 had the strongest intensity; however, these differences in staining intensity did not reach statistical significance.

We examined the relationship between the IgG subclass staining and complement (C3 and C1q) staining in renal biopsies with primary membranous glomerulonephritis. No significant association was noted between C3 staining and IgG subclass staining. However, there appears to be a positive trend between IgG3 and C1q expression; as IgG3 expression increases, so does C1q expression (Table 4; test for trend $P=0.0186$ ). Conversely, if IgG4 expression increases, C1q expression decreases $(P=0.0450)$. We did not find any statistically significant differences or associations between electron microscopy stage and C3 or C1q staining (data not shown).

\section{Discussion}

There is now evidence that, in most cases of primary/idiopathic membranous glomerulonephritis, patients have circulating anti-PLA2R IgG4 antibodies, and most, but not all patients with idiopathic membranous glomerulonephritis have IgG4-dominant subepithelial immune complex deposits, which colocalize with the PLA2R to podocytes. ${ }^{2-4}$ However, other potential antigens in primary/idiopathic membranous glomerulonephritis may also evoke an IgG4 response. ${ }^{5-8}$ Also, one has to consider that the presence of an underlying condition, that can be associated with membranous glomerulonephritis, does not necessarily exclude the possibility of a primary membranous glomerulonephritis, particularly if the glomerular capillary deposits are IgG4 dominant (eg, a person with a malignancy or hepatitis B or $\mathrm{C}$ virus infection can have membranous glomerulonephritis independent of these conditions). Our data confirmed the already known fact that, in most biopsies with primary membranous glomerulonephritis, the glomerular deposits are IgG4 dominant. However, we also found that, in very early stages (pure stage I) of membranous glomerulonephritis, the glomerular deposits are frequently IgG1 rather than IgG4 dominant. There are two possible explanations for this phenomenon. IgG1 antibodies are generally induced in response to protein antigens, whereas IgG4 antibodies are associated usually with polysaccharide antigens. ${ }^{19}$ Therefore, it is possible that in early stages of primary membranous glomerulonephritis antigens other than PLA2R have a pathogenic role, but in later stages of the disease, the antibody response to PLA2R is increasing, and an anti-PLA2R IgG4 response will become dominant. Alternately, it is theoretically possible that there is an IgG subclass switch during the progression of primary membranous glomerulonephritis. In early stages, it 
Table 2 Relationship between IgG predominance/codominance and glomerular stage in primary membranous glomerulonephritis

\begin{tabular}{|c|c|c|c|c|c|c|c|}
\hline \multirow[t]{2}{*}{$\operatorname{Ig} G$ subclass } & \multicolumn{5}{|c|}{$\begin{array}{c}\text { Electron microscopy stage } \\
n(\%) \text { predominance or codominance }\end{array}$} & \multicolumn{2}{|c|}{$\mathrm{P}$-values } \\
\hline & $1(\mathrm{n}=22)$ & $1-2(\mathrm{n}=36)$ & $2(\mathrm{n}=14)$ & $2-3(\mathrm{n}=25)$ & $3-4(\mathrm{n}=17)$ & $\begin{array}{l}\text { Predominance/ } \\
\text { codominance vs no. }{ }^{\mathrm{a}}\end{array}$ & $\begin{array}{l}\text { Test for trend } \\
(\text { pre/co vs none })^{\mathrm{b}}\end{array}$ \\
\hline $\operatorname{IgG1}$ & $14(64 \%)$ & $12(33 \%)$ & $10(71 \%)$ & $11(44 \%)$ & $6(35 \%)$ & 0.0493 & 0.3398 \\
\hline IgG2 & $0(0 \%)$ & $3(8 \%)$ & $0(0 \%)$ & $0(0 \%)$ & $0(0 \%)$ & 0.1541 & 0.4126 \\
\hline IgG3 & $5(23 \%)$ & $6(17 \%)$ & $3(21 \%)$ & $3(12 \%)$ & $3(18 \%)$ & 0.8935 & 0.5933 \\
\hline IgG4 & $12(55 \%)$ & 28 (78\%) & $13(93 \%)$ & $20(80 \%)$ & $13(76 \%)$ & 0.0964 & 0.1326 \\
\hline
\end{tabular}

${ }^{\mathrm{a}} \chi^{2}$ test comparing proportions of cases that are IgG predominant or codominant between electron microscopy stages.

${ }^{\mathrm{b}}$ Cochran-Armitage test for trend for IgG dominance across electron microscopy stage.

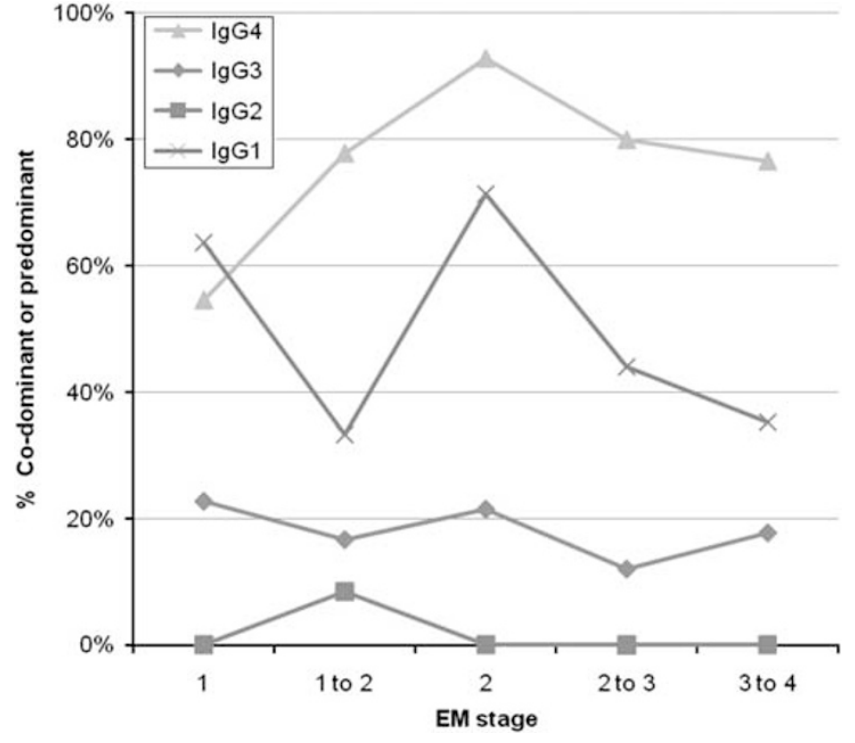

Figure 3 IgG subclass predominance or codominance in different stages of primary membranous glomerulonephritis.

is possible that there is an IgG1-dominant antiPLA2R response, which, with disease progression, will turn into an IgG4-dominant antibody response. Such IgG subclass switch has been reported in a variety of diseases, such as in malaria, ${ }^{20}$ leprosy and tuberculosis, ${ }^{21}$ and paracoccidioidomycosis. ${ }^{22}$

A Recent study by Segawa et $a 1^{23}$ from Japan, describes the IgG subclass distribution in 16 pediatric patients with 'idiopathic' membranous glomerulonephritis. These authors found that in biopsies with segmental glomerular distribution of the deposits IgG1 and IgG3 dominated in the subepithelial deposits, whereas in biopsies with global glomerular distribution of deposits IgG4 dominance was noted with deposition of other IgG subclasses as well. They hypothesized that pediatric patients with segmental glomerular capillary deposits have early stage membranous glomerulonephritis. ${ }^{23}$ If this is correct, their findings support our results in an adult patient population. Although their case number was low, Segawa et $\mathrm{l}^{23}$ also attempted to correlate the pattern
Table 3 Intensity of IgG subclass staining in different stages of primary membranous glomerulonephritis

\begin{tabular}{lccccr}
\multirow{2}{*}{$\begin{array}{l}\text { IgG } \\
\text { subclass }\end{array}$} & \multicolumn{5}{c}{$\begin{array}{c}\text { Electron microscopy stage } \\
\text { Mean } \pm \text { s.d. }\end{array}$} \\
\cline { 2 - 6 } & $\begin{array}{c}1 \\
(\mathrm{n}=22)\end{array}$ & $\begin{array}{c}1-2 \\
(\mathrm{n}=36)\end{array}$ & $\begin{array}{c}2 \\
(\mathrm{n}=14)\end{array}$ & $\begin{array}{c}2-3 \\
(\mathrm{n}=25)\end{array}$ & $\begin{array}{c}3-4 \\
(\mathrm{n}=17)\end{array}$ \\
\hline IgG1 & $2.1 \pm 0.8$ & $2.1 \pm 0.7$ & $2.4 \pm 0.6$ & $2.1 \pm 0.8$ & $2 \pm 0.8$ \\
IgG2 & $0.8 \pm 0.8$ & $0.9 \pm 0.8$ & $0.7 \pm 0.7$ & $0.5 \pm 0.6$ & $0.4 \pm 0.6$ \\
IgG3 & $1.1 \pm 1.1$ & $1.1 \pm 1$ & $1.4 \pm 0.9$ & $1.1 \pm 1$ & $1.2 \pm 1.1$ \\
IgG4 & $1.6 \pm 1.3$ & $2.2 \pm 1.1$ & $2.6 \pm 0.8$ & $2.2 \pm 1.1$ & $2.5 \pm 0.9$ \\
\hline
\end{tabular}

of glomerular complement deposition with the IgG subclasses in the glomerular deposits. They found that in membranous glomerulonephritis with segmental glomerular deposits, there was stronger C1q staining than in membranous glomerulonephritis with global glomerular deposits, indicating predominantly classical complement pathway activation in segmental (presumably early) membranous glomerulonephritis. In membranous glomerulonephritis with global deposits (but not in membranous glomerulonephritis with segmental deposits), these authors found mannose-binding lectin deposition in the glomeruli, suggesting that in most (perhaps later stages) of primary membranous glomerulonephritis, the mannose-binding lectin pathway may have a role in the continuous formation of glomerular immune complex deposits.

Our data also show that stronger glomerular staining for immunoglobulin subclasses that activate the classical pathway (such as IgG3) is associated with stronger staining for $\mathrm{C} 1 \mathrm{q}$, and that there is a negative correlation between the staining intensity for IgG4 and $\mathrm{C} 1 \mathrm{q}$ in the glomerular deposits in membranous glomerulonephritis. However, the routine direct immunofluorescence method, used in the diagnostic workup of renal biopsies, has poor sensitivity and is semiquantitative; therefore, solid conclusions cannot be drawn. We only used anti-C1q and anti-C3 antibodies because these antibodies are part of the routine renal biopsy workup (this study is based on routine renal biopsy 
Table 4 Relationship between IgG subclass and C1q staining in primary membranous glomerulonephritis

\begin{tabular}{|c|c|c|c|c|c|c|c|c|}
\hline \multirow[t]{2}{*}{ IgG subclass } & \multicolumn{3}{|c|}{$\begin{array}{c}\text { C1q } \\
\text { Mean } \pm \text { s.d. IgG Score }\end{array}$} & \multicolumn{3}{|c|}{$\begin{array}{c}\text { C1q } \\
\operatorname{Ig} G \mathrm{~N}(\%) \text { positive expression }\end{array}$} & \multicolumn{2}{|c|}{$\mathrm{P}$-value } \\
\hline & $0(\mathrm{n}=88)$ & $1(\mathrm{n}=17)$ & 2 or $3(\mathrm{n}=5)$ & $0(\mathrm{n}=88)$ & $1(\mathrm{n}=17)$ & 2 or $3(\mathrm{n}=5)$ & Yes/no positivity & Test for trend ${ }^{\mathrm{b}}$ \\
\hline IgG1 & $2 \pm 0.8$ & $2.3 \pm 0.7$ & $2.3 \pm 0.6$ & $84(95 \%)$ & $17(100 \%)$ & $5(100 \%)$ & 1.0000 & 0.5107 \\
\hline IgG2 & $0.6 \pm 0.7$ & $0.9 \pm 0.7$ & $1.2 \pm 0.9$ & 43 (49\%) & $12(71 \%)$ & $4(80 \%)$ & 0.1444 & 0.0604 \\
\hline IgG3 & $1 \pm 0.9$ & $1.6 \pm 1.1$ & $1.9 \pm 0.7$ & $52(59 \%)$ & $14(82 \%)$ & $5(100 \%)$ & 0.0487 & 0.0186 \\
\hline IgG4 & $2.4 \pm 1.1$ & $1.6 \pm 1.2$ & $1.3 \pm 1.2$ & $81(92 \%)$ & $14(82 \%)$ & $3(60 \%)$ & 0.0445 & 0.0450 \\
\hline
\end{tabular}

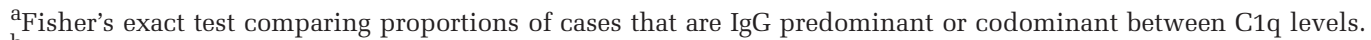

${ }^{\mathrm{b}}$ Cochran-Armitage test for trend for IgG dominance across C1q positivity.

material), and because the objective of the study was the analysis of IgG subclass distribution and not the morphological dissection of the complement cascade. Although there is no doubt that the complement system has a role in human and experimental membranous glomerulonephritis, ${ }^{24-26}$ our knowledge about the exact role of complement activation in the pathogenesis of membranous glomerulonephritis is scant. It appears that the complement system may have different roles in various forms and, perhaps, even in various stages of membranous glomerulonephritis. Detailed studies addressing the role of the complement system in the pathogenesis of membranous glomerulonephritis will be relevant because the results could have important therapeutic implications.

In summary, our findings indicate a heterogeneous immune response in primary membranous glomerulonephritis. One has to consider that PLA2R is not the only antigen relevant in the pathogenesis, particularly in early stage of the disease. Routine immunofluorescence studies used in renal biopsy evaluation are only semiquantitative and relatively insensitive. Therefore, it would be relevant to conduct studies for the potential target antigens in different stages of primary membranous glomerulonephritis, particularly in early stage (glomerular stage I).

\section{Disclosure/conflict of interest}

The authors declare no conflict of interest.

\section{References}

1 Schwartz M. Membranous glomerulonephritis, In: Jennette JC, Olson JL, Schwartz MM, Silva FG(eds) Heptinstall's Pathology of the Kidney, 6th edn. Lippincott Williams \& Wilkins: Philadelphia; 2007, pp 205-251.

2 Beck LH Jr, Bonegio RG, Lambeau G, et al. M-type phospholipase A2 receptor as target antigen in idiopathic membranous nephropathy. $N$ Engl J Med 2009;361:11-21.
3 Hofstra JM, Beck LH Jr, Beck DM, et al. Antiphospholipase A2 receptor antibodies correlate with clinical status in idiopathic membranous nephropathy. Clin J Am Soc Nephrol 2011;6:1286-1291.

4 Qin W, Beck LH Jr, Zeng C, et al. Anti-phospholipase A2 receptor antibody in membranous nephropathy. J Am Soc Nephrol 2011;22:1137-1143.

5 Prunotto M, Carnevali ML, Candiano G, et al. Autoimmunity in membranous nephropathy targets aldose reductase and SOD2. J Am Soc Nephrol 2010;21: 507-519.

6 Bruschi M, Ml Carnevali, Murtas C, et al. Direct characterization of target podocyte antigens and autoantibodies in human membranous glomerulonephritis: Alpha-enolase and borderline antigens. J Proteomics 2011;74:2008-2017.

7 Debiec H, Lefeu F, Kemper MJ, et al. Early-childhood membranous nephropathy due to cationic bovine serum albumin. N Engl J Med 0211;364:2101-10.

8 Murtas C, Bruschi M, Candiano G, et al. Coexistence of different circulating anti-podocyte antibodies in membranous nephropathy. Clin J Am Soc Nephrol 2012;7: 1394-1400.

9 Doi T, Mayumi M, Kanatsu K, et al. Distribution of IgG subclasses in membranous nephropathy. Clin Exp Immunol 1984;58:57-62.

10 Noel LH, Aucouturier P, Monteiro RC, et al. Glomerular and serum immunoglobulin $G$ subclasses in membranous nephropathy and anti-glomerular basement membrane nephritis. Clin Immunol Immunopathol 1988;46:186-194.

11 Haas M. IgG subclass deposits in glomeruli of lupus and nonlupus membranous nephropathies. Am J Kidney Dis 1994;23:358-364.

12 Kuroki A, Shibata T, Honda H, et al. Glomerular and serum IgG subclasses in diffuse proliferative lupus nephritis, membranous lupus nephritis, and idiopathic membranous nephropathy. Intern Med 2002; 41:936-942.

13 Kearney N, Podolak J, Matsumura L, et al. Patterns of IgG subclass deposits in membranous glomerulonephritis in renal allografts. Transplant Proc 2011;43: 3743-3746.

14 Ohtani H, Wakui H, Komatsuda A, et al. Distribution of glomerular IgG subclass deposits in malignancyassociated membranous nephropathy. Nephrol Dial Transplant 2004;19:574-579.

15 Omokawa A, Komatsuda A, Nara M, et al. Distribution of glomerular IgG subclass deposits in patients with membranous nephropathy and anti-U1 
ribonucleoprotein antibody. Nephrol Dial Transplant 2012;27:1937-1941.

$16 \mathrm{Qu} \mathrm{Z}$, Liu G, Wu LH, et al. Absence of glomerular IgG4 deposition in patients with membranous nephropathy may indicate malignancy. Nephrol Dial Transplant 2012;27:1931-1937.

17 Ehrenreich T, Chung J. Pathology of membranous nephropathy. Pathol Annu 1968;3:145-186.

18 Satoskar AA, Brodsky SV, Nadasdy G, et al. Discrepancies in glomerular and tubulointerstitial/vascular immune complex IgG subclasses in lupus nephritis. Lupus 2011;20:1396-1403.

19 Schroeder H, Cavacini L. Structure and function of immunoglobulins. J Allergy Clin Immunol 2010; 125:S41-S52.

20 Tongren JE, Drakeley CJ, McDonald SL, et al. Target antigen, age, and duration of antigen exposure independently regulate immunoglobulin $G$ subclass switching in malaria. Infect Immun 2006;74:257-264.

21 Sousa AO, Henry S, Maroja FM, et al. IgG subclass distribution of antibody responses to protein and polysaccharide mycobacterial antigens in leprosy and tuberculosis patients. Clin Exp Immunol 1998;111: 48-55.

22 Baida H, Biselli PJC, Juvenale M, et al. Differential antibody isotype expression to the major Paracoccidioides brasiliensis antigen in juvenile and adult form paracoccidioidomycosis. Microbes Infect 1999;1:273-278.

23 Segawa Y, Hisano S, Matsushita $M$, et al. IgG subclasses and complement pathway in segmental and global membranous nephropathy. Pediatr Nephrol 2010;25:1091-1099.

24 Schulze M, Donadio JV Jr., Pruchno CJ, et al. Elevated urinary excretion of the C5b-9 complex in membranous nephropathy. Kidney Int 1991;40:533-538.

25 Couser WG, Schulze M, Pruchno CJ. Role of C5b-9 in experimental membranous nephropathy. Nephrol Dial Transplant 1992;7(Suppl 1):25-31.

26 Kon SP, Coupes B, Short CD, et al. Urinary C5b-9 excretion and clinical course in idiopathic human membranous nephropathy. Kidney Int 1995;48: 1953-1958. 